International Journal of Network Security \& Its Applications (IJNSA), Vol.5, No.1, January 2013

\title{
AN INTEGRATED SOLUTION FOR BOTH MONITORING AND CONTROLLING FOR AUTOMIZATION USING WIRELESS SENSOR NETWORKS: A CASE STUDY
}

\author{
${ }^{1}$ M Gnana Seelan and Ch A S Murty ${ }^{2}$ \\ ${ }^{1}$ Centre for Development of Advanced Computing, Hyderabad, India \\ mgseelan@cdac.in \\ ${ }^{2}$ Centre for Development of Advanced Computing, Hyderabad, India \\ chasmurtyecdac.in
}

\begin{abstract}
Temperature monitoring plays a major role in controlling it according to its varied conditions. This process is common in all critical areas like data centre, server rooms, grid rooms and other data communication equipped rooms. This is mandatory for each organization/industry to impart such process, as most of the critical data would be in data centre along with their network infrastructure which having various electronic, electrical and mechanical devices are involved for data transmissions. These devices are very much depend on the environmental factors such as temperature, moisture, humidity etc., and also emit heat in the form of thermal energy when they are in functional. To overcome these heats, the server/data centre room(s) would be engaged with multiple (distributed) air-conditioning (ac) systems to provide cooling environment and maintain the temperature level of the room. The proposed paper is the study of automization of monitoring and controlling temperature as per desired requirements with wsn network
\end{abstract}

\section{KEYWORDS}

WSN, Distributed AC, Temperature Control, Blue-tooth, IR circuit, Temperature Monitoring, Data and Grid room, HVAC

\section{INTRODUCTION}

In the recent years, new technologies are evolved on automization of various processes. With that we understood that, introducing automatic controls in terms of temperature monitoring and control using wired or wireless devices could be the best way to reduce the human interaction and human prone errors for controlling the systems. In addition there is a necessity to maintain control level for distributed systems.

Data centre or High performance computing labs such as Grid, Cluster or Server rooms are identified as critical Infrastructure of an organization. There is a need to maintain stable temperature for secure the data communication, secure from various physical damages with respect to various environmental threats. So, there is necessity to have a temperature control and monitoring system to maintain the stability of room temperature in the Data centre to increase productivity in-terms of life of various electrical and electronic components. However, the monitoring and controlling temperature with in Data centre is very difficult as the flow of HVAC conditions are depended as per requirements of components in the Data centre and the requirements are different to different components and varies time to time [1]. Whenever the temperature exceeds the limit of threshold levels, many horrendous events can occur, such as 
International Journal of Network Security \& Its Applications (IJNSA), Vol.5, No.1, January 2013

server meltdown, loss of data and even fire may occur and may expect huge loss in terms of life, damages etc.,. These events also cost time and money to replace/repair damaged goods [2].

\subsection{Motivation}

The monitoring and controlling various temperature levels for various components with in data centre would be difficult in manual monitoring and controlling as 24X7 basis and it would be better to have facility such as automization of such process on each minute and each second if possible. There are automatic controls for monitoring and controlling separately available for centralized systems and by keeping in view of distributed components, it would be required to have a combined automatic monitoring and controlling network for data centres. Temperature controls are commonly used in the world to maintain the temperature at certain level or ranges. There would be a seamless technology which can sense the temperatures on each second and compare with desired requirements and also inform to the automatic controls to control as per desired temperatures [3].

The main aim of the paper is to suggest and propose a low cost wireless sensor network with a low power IR remote sensor to control the distributed air conditioning system and integrated with automatic monitoring system. The main functions of the proposed system are: Continuous room temperature Monitor and Maintenance; to transmit signal to the server through receiver; Continuous controlling the distributed air conditioning system with respect to the temperature [4].

There are popular solutions for Automatic temperature monitoring and may be done through manually controlling the same by observing automatic temperature levels. But along with automatic monitoring, there is need for automatic controlling in distributed environment such as distributed Air Conditioning systems. This paper is discussing about the implementation of both automatic monitoring and controlling solutions as an integrated solution for monitoring and controlling various components through WSN networks [5].

\section{RELATED WORK}

Globally, number of WSN solutions is available for automization of temperature monitoring. But no solutions found for distributed systems. In view of above problem, we need a solution which could perform monitoring and instruct controller to control automatically as per required desired temperature levels. But we could not found a unique integrated solution which could perform both. From the literature survey for temperature control system and monitoring system some of the researchers and scientist mentioned as below.

The key motivation is from the Wireless Sensor Networks Research Group and is formed by researches and developers team throughout the world. Squid-Bee is an open-source WSN platform, where each Squid-Bee Mote takes environmental parameters with its three sensors (humidity, temperature and lightness) and sends through WSN [6].

Sridevi Veerasingam, Saurabh Karodi, Sapna Shukla and Mehar Chaitanya Yeleti on "Design of Wireless Sensor Network node on ZigBee for Temperature Monitoring" described the function of the wireless data logging system [7].

In continuation to the data logger the work by Ahmad Faris Bin Zulkifli on"Automatic Room Temperature Control with Security System" highlighted the importance of automated temperature monitoring of a room and controlling with automated mechanism [8].

The work by Vongsagon Boonsawat, Jurarat Ekchamanonta and Kulwadee Bumrungkhet on "XBee Wireless Sensor Networks for Temperature Monitoring " presented the function an embedded WS capable of monitoring \& managing $\mathrm{N}$ prototype system for temperature monitoring in a building [9]. 
International Journal of Network Security \& Its Applications (IJNSA), Vol.5, No.1, January 2013

Over the past, many WSN systems have been developed and studied for numerous applications. On this regard lot of study been done to support the work and propose an integrated automatic temperature monitoring and controlling system.

\section{MethodologY}

The details of the system explained in the subsequent points.

\subsection{System Overview}

The experimental integrated monitoring and controlling system is shown in Figure: 1. consists of (a) Temperature Monitoring System (TMS): (b) Temperature Controlling System (TCS): (c) Comparator and a Gateway

Figure: 1 Experimental Setup

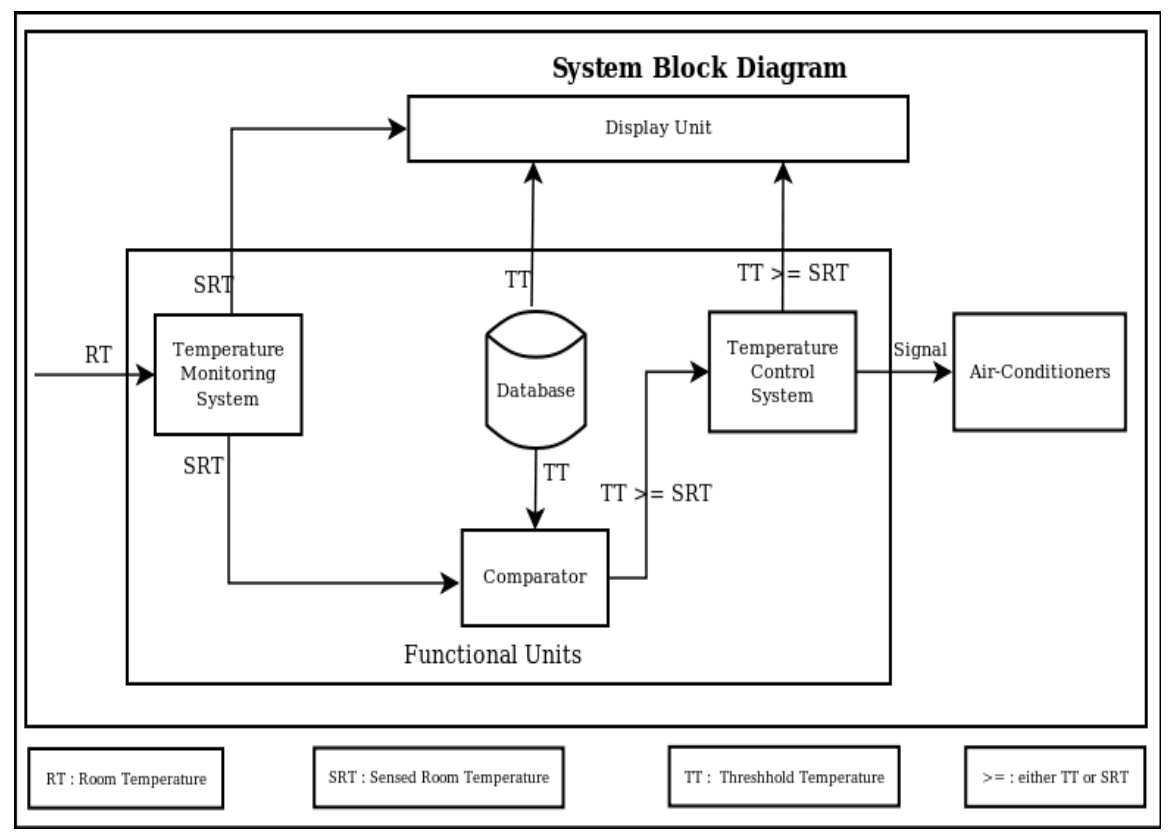

Temperature Monitoring System contains four parts as (i) Client nodes which sense the surrounding temperature with respect to each nodes and wait the response from (ii) receiver which collects the sensing data from different sensors (client nodes) and forwards the same to (iii) the database server component which would be used stores the data and display using web user interface (iv) web server component for reports.

Temperature Controller System contains three components as (i) IR Remote connected to (ii) a computer which controls (iii) Air Conditioning (AC system) through Infrared communication.

Comparator is having a web application to compare the threshold temperature (TT) with the sensing temperature (SRT). Serial Gateway of TMS is generally used for connecting receiver node with the database server.

\subsection{Design and Development}

The integrated temperature monitoring and controlling system has three major components as explained in the system overview. The Major components has been further divided as subsystem levels and been explained separately in the succeeding paragraphs. 


\subsubsection{Temperature Monitoring System:}

The detailed discussion of the monitoring system is shown in the below figure.

Figure: 2 Experimental Temperatures Monitoring System

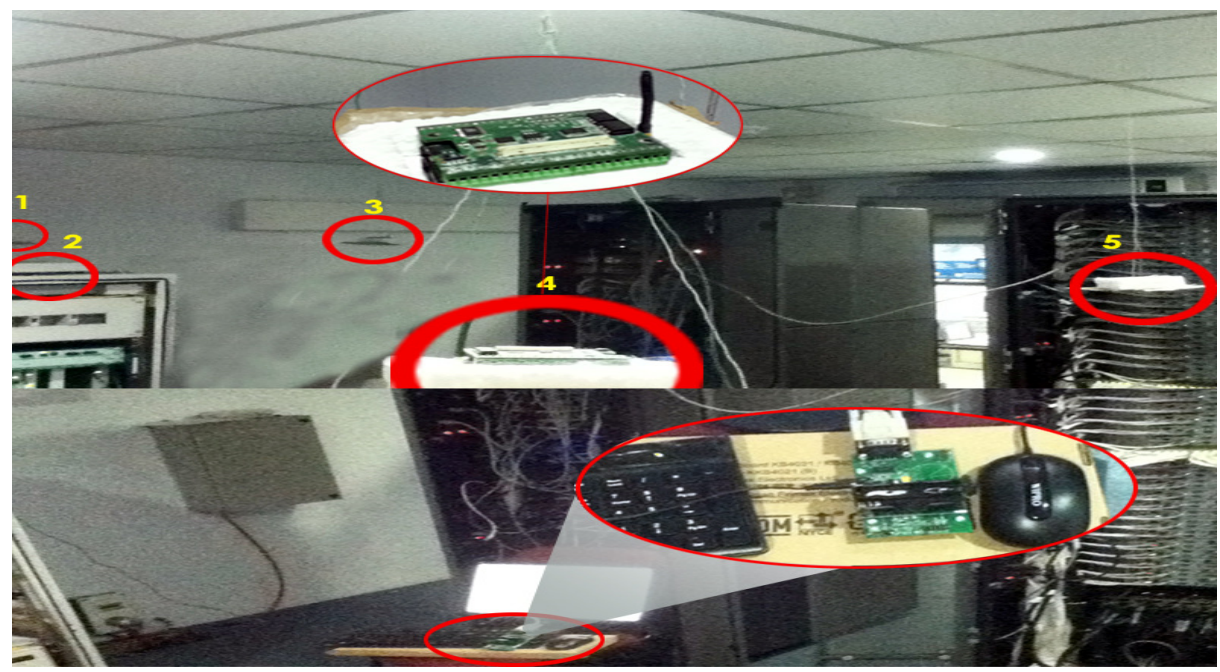

The experimental temperature monitoring system and its components are shown in Figure: 2 Temperature Monitoring System contains four parts as: (i) Client sensor nodes: (ii) Receiver node (Server node): (iii) The database server component: and (iv) Web server component

Each of the subsystem explained briefly in the succeeding sections.

\subsubsection{Client Sensor nodes}

The sensors (nodes) convert the analog temperature signal in terms of volts into degree Celsius according to the value. The client (sensor) node collecting the data through sensing technology and try to connect the server to upload as the same as immediately because of there is no storage space. Client sensor which senses the surrounding temperature with respect to each node and wait the response from centrally placed receiver node. Wireless sensor network usually consists of a large number of nodes those are deployed in the sensing area and are equipped with different kinds of sensing, computation and communication units. These functional units enable WSN nodes to cooperatively collect, process, and transmit information to the receiver [4].

Client values with respect to the surrounding room temperature values are to minimize the break down in the cluster room.

$$
\begin{aligned}
& \text { 1. Node1 Value }=20: 35 \mathrm{C} \\
& \text { 2. } \text { Node2 Value }=22: 15 \mathrm{C} \\
& \text { 3. Node3 Value }=23: 45 \mathrm{C} \\
& \text { 4. Node4 Value }=26: 35 \mathrm{C} \& \\
& \text { 5. Node5 Value }=30: 35 \mathrm{C}
\end{aligned}
$$

\subsubsection{Receiver node (server node)}

Receiver node (Server node) normally placed in the central location in a way to function using star topology. The function of the server node is to collects the sensing data from different sensors (client nodes) and forwards the same to the database server for updating the values with respect to the client nodes shown in Fig: 1. Receiver will respond to the all clients for updating the data. 
International Journal of Network Security \& Its Applications (IJNSA), Vol.5, No.1, January 2013

\subsubsection{Database server Component}

Database server major function is to store each and every data received from the receiver. The database server also responsible for storing the thresh-hold Table: 1 temperature of all the nodes.

Table:1 Thresh-hold Temperature for Room

\begin{tabular}{|l|c|c|c|c|c|}
\hline Threshold & Node1 & Node2 & Node3 & Node4 & Node5 \\
\hline Minimum & 17 & 18 & 22 & 22 & 25 \\
\hline Maximum & 23 & 25 & 27 & 28 & 32 \\
\hline
\end{tabular}

The data collected from the server node will be stored in the mysql/postgres/Sqlite database. We are using MySQL database for storing the data.

\subsubsection{Web server component}

In addition to the management of data there is a need to create reports of the collected data/output to analyse its performance, by web interface. To have web interface web server is required. We are using php (lampp) as a whole for hosting and retrieving data through web interface. The stored data would be displayed using php web user interface. The web servers other responsibilities are providing user friendly interface by which all the clients nodes temperature could be monitored, and could be utilized to create reports in the form of html etc.,.

\subsubsection{Temperature Controlling System}

Temperature controlling system has the major functionality of controlling AC with the usage of infrared remote. Infrared remote sends the infra-red signal to the AC's as per controllers' instruction.

Table: 2 Setting for AC- No load on the Cluster

\begin{tabular}{|c|c|c|c|c|c|}
\hline Values & AC-1 & AC-2 & AC-3 & AC-4 & AC-5 \\
\hline Minimum & 23 & 23 & 22 & 21 & 20 \\
\hline Maximum & 24 & 25 & 23 & 22 & 21 \\
\hline
\end{tabular}

AC will set the value Table: 2 with respect to the received infrared signal. In general, ACs is set with a range of values assigned to the voltage.

Figure: 3 Experimental Temperature Control system

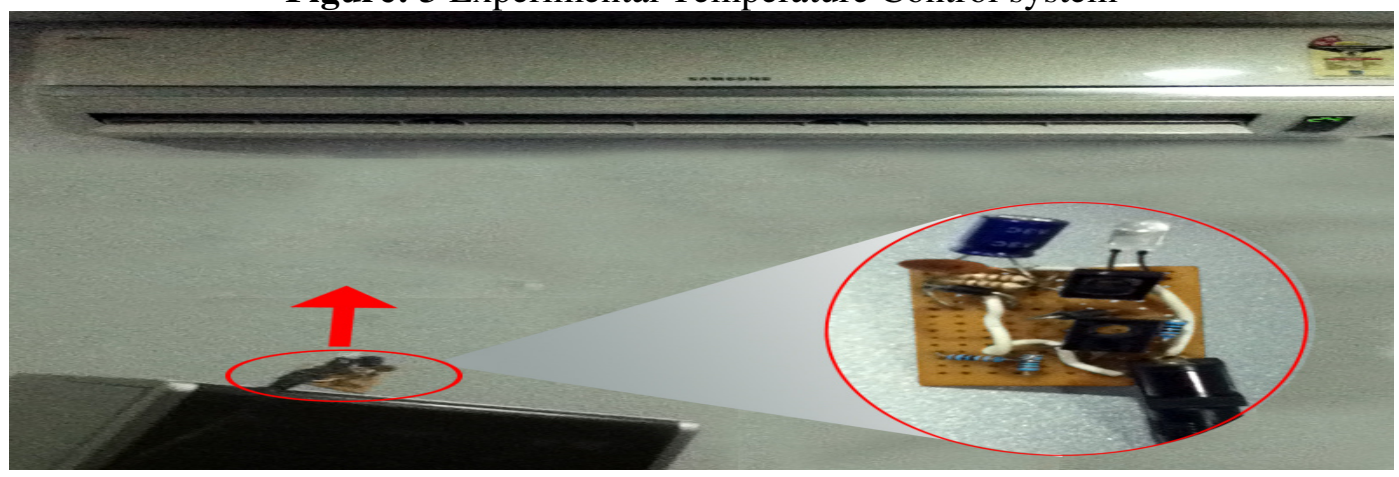


International Journal of Network Security \& Its Applications (IJNSA), Vol.5, No.1, January 2013

Figure: 3. Demonstrate the practical implementation of temperature control system. Temperature Controller System contains three components as: (i) IR Remote circuit: (ii) Computer with control application: (iii) Air Conditioning (AC system)

\subsubsection{IR Remote circuit}

Infrared mechanism will control as getting instruction from the controller. IR will send signal to the $\mathrm{AC}$ for respective values [11].

\subsubsection{Computer with control application}

To system is installed with web application using php (lampp) as a whole for hosting and retrieving data through web interface. The stored data would be displayed using php web user interface. The web servers other responsibilities are providing user friendly interface by which all the clients nodes temperature could be monitored, and could be utilized to create reports in the form of html etc.,.

\subsubsection{Air Conditioning (AC system)}

The AC are deployed all over the room object is to control and stabilize the temperature inside the room.

\subsubsection{Comparator and a Gateway}

The comparator is an application configured in the monitoring system. The function of the application is to compare the sensed value collected by the receiver with the thresh-hold values of each node.

Figure: 4 Experimental Temperature Test Condition

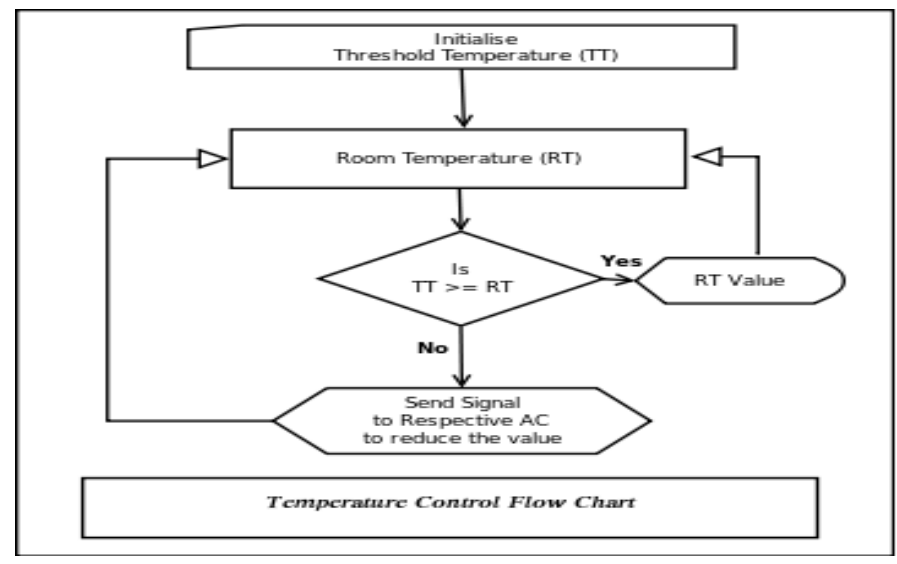

There is a need to automate the comparison process by the comparator. The Serial gateway transmits the received data from the client nodes to the database server through serial interface. For experimental setup we used MySQL database to store the data's of each nodes and threshhold temperature. Comparator is having a web application to compare the threshold temperature (TT) with the sensed room temperature (SRT). Serial Gateway of TMS is generally used for connecting receiver node.

\section{SYSTEM SPECIFICATION}

The system is considered as closed loop control system. Compared with the existing temperature monitoring and controlling system, this paper proposes an integrated solution based 
International Journal of Network Security \& Its Applications (IJNSA), Vol.5, No.1, January 2013

on wireless sensor network technology. This approach is to propose an automated \& costeffective real-time in-house solution for high performance computing environment. The proposed system could communicate with individual nodes providing automated control to the distributed system.

Network Specification: The architecture is of star topology, receiver in the center to process collected data's of client nodes. It gathers sensor readings from all the client nodes.

Hardware Specification: A WSN node consists of some sensors as client and receiver, communication modules, Infrared Remote circuit, and a computer system for running application. Sensors: Temperature sensors are having MICAz (It is a $2.4 \mathrm{GHz}$ Mote module used for enabling low-power, wireless sensor networks).The sensors support reliable wireless sensor network platform and ad-hoc mesh networking. They support tinyOS operating system and also provide reliability [12].

Other important components for the implementation of the systems are: Wireless Sensor Networking and Infra-red with its functionalities; for the future work Knowhow to develop applications using Blue-tooth [13] and Serial device, Blue-tooth and TCP Socket Programming on GNU/Linux and AVR Firmware Programming.

Software Specification: Web-Server: We used html for web interface in the Centralized computer system. Database: We used MySQL server to run on the computer. The computer has LAMPP web server for Query MySQL command with PHP language accordingly.

Implemented System Limitation: As we are used multiple components for the experiment, there are various parameters need to be considered. The distance between the clients nodes to the receiver are very narrow. Also there would be many line of site difference may occur between the nodes. The nodes may get over heated and send junk data to the receiver and which may collapse entire setup.

\section{SYSTEM IMPLEMENTATION}

By considering 200 Square Feet area as pilot basis for experimental result, we implemented a prototype system of automated Temperature monitoring and control system based on Wireless Sensor Network (WSN). Although there are many wireless solutions as discussed in the Related Work section for monitoring or controlling, this system would be the solution for the distributed systems as shown in the figures above each system separately. It also satisfied the wireless communication standard specifications, and adapted to any data Centre and grid room with its extensive characteristics.

Considering the above, we deployed WSN system in a room which is having 40 node clusters and also not having proper air conditioning system. The system is designed in a way to monitor the stable temperature and control the air conditioning system for Clusters.

The Sensors (Client nodes) are placed in five different locations:

- Node1 placed 5 meters from the back-end of the server rack

- Node2 placed 4 meters from the back-end of the server rack

- Node3 placed 2 meters besides from the server rack

- Node4 placed 1 meter from the front-side of the server rack

- Node5 placed half a meter from the back-end of the server rack

Monitoring the sensitive data for various temperature changes due to exhaust of heat from physical nodes of cluster, the temperature varies if the jobs increase in the cluster. 
International Journal of Network Security \& Its Applications (IJNSA), Vol.5, No.1, January 2013

The temperature inside the cluster room is always high and unstable and the human management was failed to control the temperature manually. Hence we proposed an automatic system is required to control the temperature within the cluster room by utilizing the temperature sensors.

\section{TESTING}

We setup prototype with WSN supported in a room which has 40 node cluster, storage and other related devices. The actual control process is shown in the Fig: 2 explain about the test conditions. Individual test has been carried out with each component before integrating as a whole. The test has been done with placing the sensors nodes in different places of the specific room as discussed in the above section. The room was deployed with 5 different A/Cs.

The desired temperature for the normal function of the cluster should be maintained between $10 \mathrm{C}(50 \mathrm{~F})$ to $28(82)$ as per the load. Mostly all components such as computing nodes, and other networking equipment are designed to operate within a moderate narrow temperature ranges. To ensure reliable operation and the long life for components, we should ensure that the temperature stays within the limited band.

In this reason, there is a need to introduce laws of thermodynamics, as we are proposing a solution for temperature control which is normally in the form of heat energy. It is from first law of thermodynamics the energy produced by a machine is proportional to the heat dissipation by the machine. "In a thermodynamic process, the increment in the internal energy of a system is equal to the difference between the increment of heat accumulated by the system and the increment of work done by it "[14].

Likewise water flowing down-hill, as heat energy will naturally and automatically move from a hot place to a cooler place. There is a need to maintain a temperature level for any component to function properly. The set temperature is called as thresh-hold temperature (TT). Each client node has different values with respect to the distance from the cluster. The threshold temperature range is from 18 to 28 as the value will increase if the clients are placed near to the exhaust out of heat from the clusters. The temperatures vary from front side to backside of the clusters in general.

The each mote senses the respective surrounding temperature, and sends to the server mote to store in the computer server. The collected sensed room temperature (SRT) need to compare with respective threshold temperature (TT). If the room temperature is less than or equal to the threshold then no action will be taken, if the temperature is greater than the threshold then a signal through IR will be sent to the respective air conditioner to change accordingly.

\subsection{Component Testing}

Every sensor is tested individually with the data error. It has been observed that whenever the temperature of the data centre increases it also affects the sensors. As the sensors are set with a functional voltage range. The receiver node will overload if all clients request receiver to upload the collected data.

Experimental Temperature measurements: As from law of thermodynamics explained in the above section it is understood that, heat energy is directly proportional to temperature, and also the following points are noted for the consideration while testing:

1. The distance from the cluster - placement of sensor nodes (clients).

2. The direction of the cluster with respect to the clients.

3. Start \& end time of the jobs in the cluster.

4. Number of nodes utilized for running the job. 
International Journal of Network Security \& Its Applications (IJNSA), Vol.5, No.1, January 2013

5. Distance from the AC, client nodes as well as IR remote.

6. Line of site of the client nodes with the receiver node.

We performed an experimental filed test for the temperature monitoring in the cluster room between 08:30 to 20:00.

The parameters considered to explain the scenarios:

1. Temperature as (T) \& Room Temperature as (RT)

2. Heat dissipation (Heat Energy) as (HE) \& Error free Temperature as (EFT)

3. Sensed Room Temperature as (RST) \& Sensor Temperature as (ST)

$$
\text { Hence EFT = SRT-ST }
$$

The experiment was carried out for automization and manual control of the temperature control. For this we have taken reading and analysed the data from the database are considered for scenario-1 (Cluster with no jobs), Scenario-2 (after the jobs started in the cluster) and scenario-3 (Cluster with full load). At the end we have explained the reason for the difference in the values.

As part of experiment, the client nodes are set to update the database for every 2 Minutes interval with the data. The updated data in the database was so huge, it is difficult to compile and compare. Hence we considered the data for every 30 Minutes interval for this paper from the database.

Normally the temperature varies inside the cluster room because of the heat emission of each server in the cluster as discussed above from law of thermodynamic. We observed that front side of the server is cooler than backside of the server node.

Scenario-1: Cluster with no load: The diagram for the cluster no loads (no jobs) running on the cluster shown in the Figure: 5.We also set the AC values in Normal to stable the room temperature and the details is shown in the Table: 2 for the reference.

Figure: 5 Cluster with no load

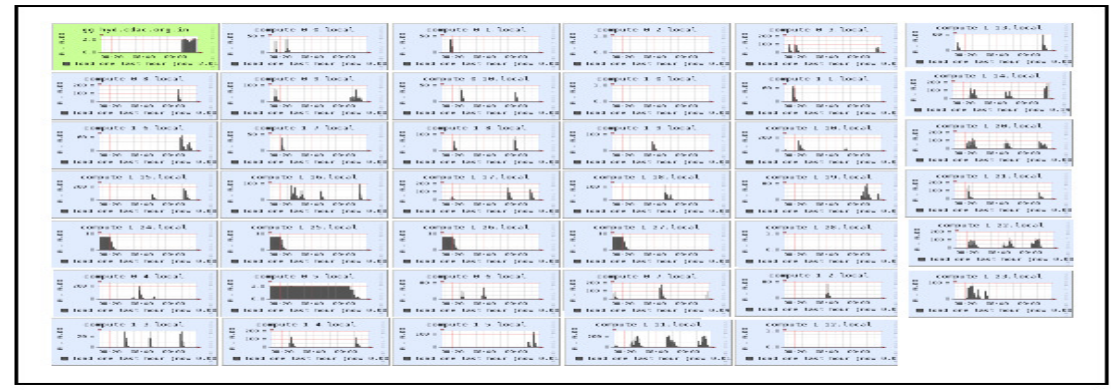

As the nodes are up and there were no jobs running hence the room is normal and observation.

1. The readings were taken from 08:30 to 19:30 Hrs, for all five nodes.

2. The values of each node varied with respect to time and no relation to each other.

3. The peak value is observed at 16:00 Hrs from Node5 with 25:40C

4. The following observations for each nod were recorded as below:

- Node1: The minimal \& maximum value observed as 18:15C \& 19:50C.

- Node2: The minimal \& maximum value observed as 19:25C \& 21:30C. 
International Journal of Network Security \& Its Applications (IJNSA), Vol.5, No.1, January 2013

- Node3: The minimal \& maximum value observed as 19:05C \& 21:45C.

- $\quad$ Node4: The minimal \& maximum value observed as 19:25C \& 21:45C.

- Node5: The minimal \& maximum value observed as 20:40C \& 25:40C.

Graph: 1 Cluster with Full load

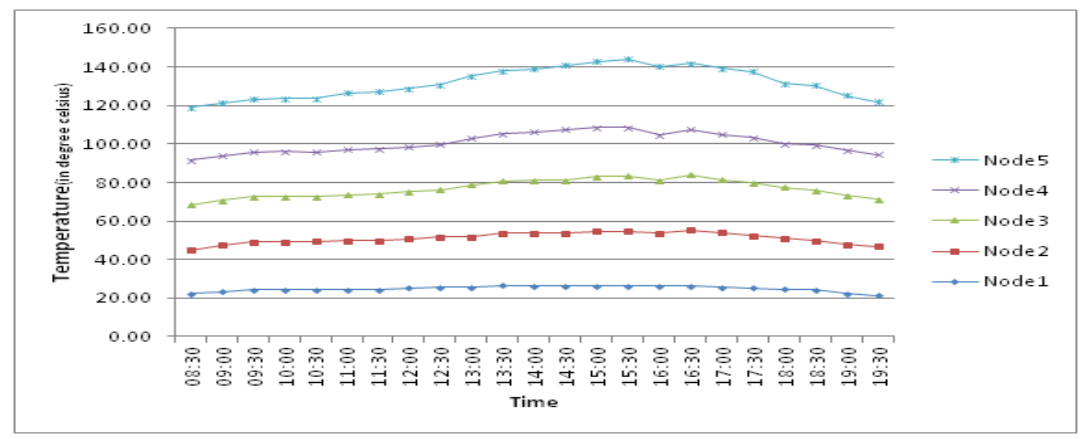

The above experimental output explains clearly that the temperature depends on the following:

1. Continuous running of server increases the temperature and to manage the same requires manual adjustment of A/C's.

2. Temperature increases if no proper air conditioning facilities in the cluster room.

3. Temperature increases with the environmental heat such as external heats.

4. Temperature also increases according to the increase in the human intervention or movements in the cluster room.

5. The minimal value is from Node1 and the maximum value is from Node5.

The results of variation in temperature levels with respect to the client with time are shown in Graph: 1 .

Scenario-2: Cluster with Start of load: The Figure: 6 shows Initial stage of the cluster when jobs are started on the cluster.

Figure: 6 Cluster with Start of load

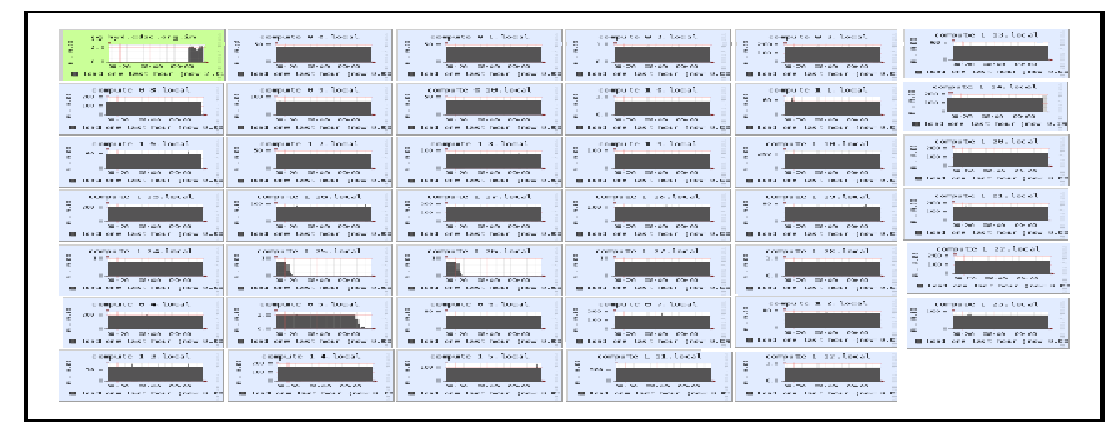

The second scenario was tested with running number of jobs full load with running jobs in the cluster nodes. The reading has been considered after changing the $\mathrm{AC}$ values in each of the respective client nodes. If we adjust the ac values when temperature rises then it reduces and stabilizes. The experimental output shows that, whenever manual change is done it takes time as because of the following reasons:

Every time he/she has to set the value to the AC's. It may not worth full because the rise of temperature will not be slow. Human body is not addicted with high temperature situation; 
International Journal of Network Security \& Its Applications (IJNSA), Vol.5, No.1, January 2013

sometimes there could be sudden as well abnormal rise in the temperature. During the period human cannot control the same, which would lead to damage.

Graph: 2 Cluster with Full load

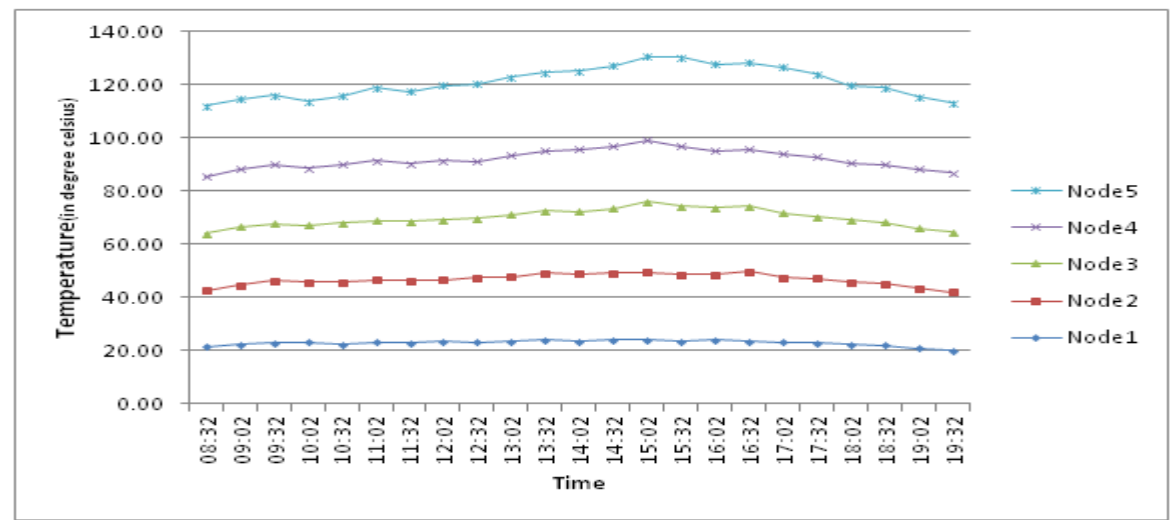

1. The readings were available from 08:32 to 19:32 Hrs, for all five nodes.

2. The peak value is observed at 17:02 Hrs from Node5 with 41:50C. and

3. The observation for each node separately noted after the start of the jobs in the cluster, the brief explanation for each node is given in the subsequent points:

- Node1: The minimal \& maximum value observed as 21:10C \& 24:25C.

- Node2: The minimal \& maximum value observed as 21:35C \& 26:35C

- Node3: The minimal \& maximum value observed as 22:15C \& 29:55C.

- Node4: The minimal \& maximum value observed as 24:25C \& 29:55C.

- Node5: The minimal \& maximum value observed as 29:10C \& 41:50C.

As from above observation it is clear that the temperature increase with the load in the cluster and huge difference between minimum and maximum.

The results of variation in temperature levels with respect to the client with time are shown in Graph:2.

Scenario-3: Cluster with Full load: The observation has been taken after the cluster with full load and is shown in the Figure: 7.

Figure: 7 Cluster with Full load

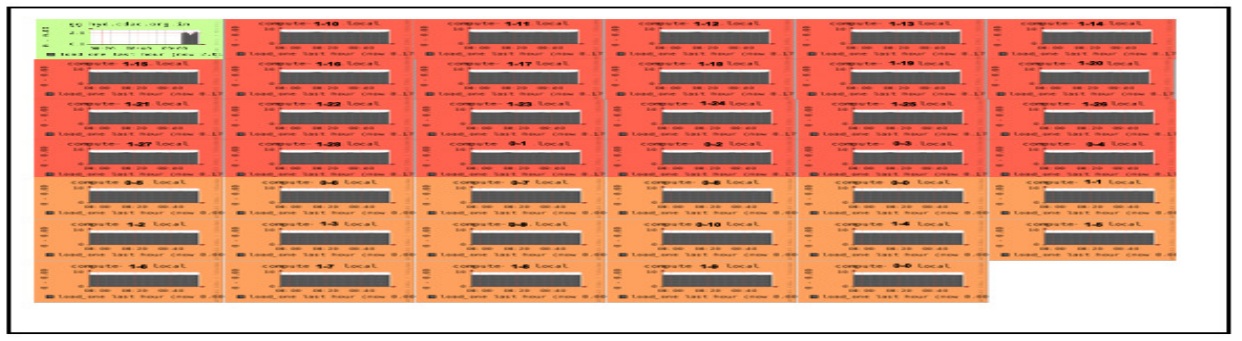

1. The readings were available from 08:50 to 19:30 Hrs, for all five nodes and was considered every 45 Minute / One hour with the condition. 
International Journal of Network Security \& Its Applications (IJNSA), Vol.5, No.1, January 2013

2. The peak value is observed at 17:02 Hrs from Node5 with 41:50C. and

3. The observation for each node separately noted after the start of the jobs in the cluster, the brief explanation for each node is given in the subsequent points:

Time delay for the control: Form above observation it was understood that controlling the AC by manual (human) control with respect to automatic control is having huge difference. We observed that, to control each node each second there is a need to monitor the deference in the temperature and other parameters. Human involvement will increase and slow the processes.

The formula for calculating the rise with respect to temperature is explained below.

Graph: 3 Cluster with Full load

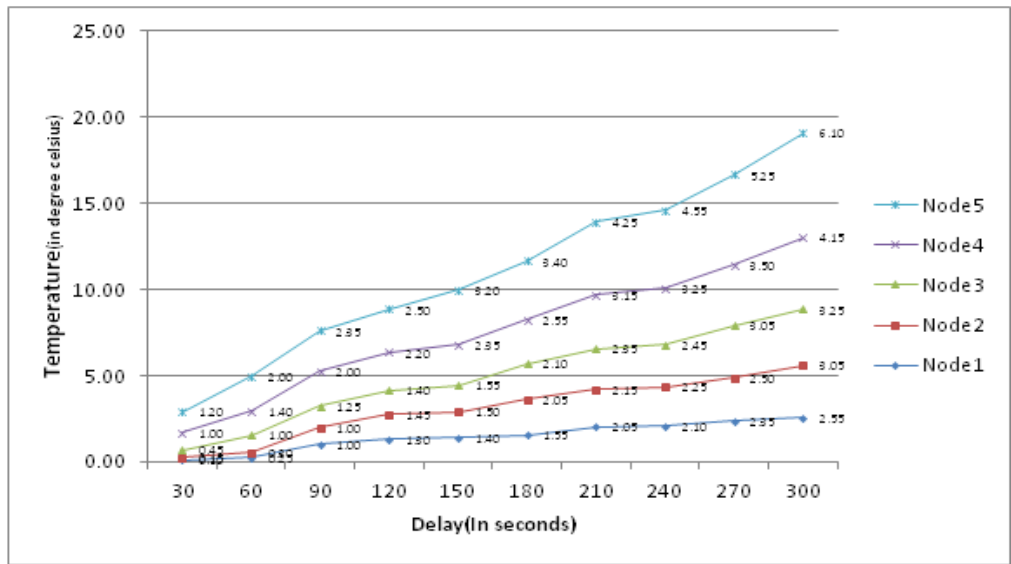

From the Graph: 3 delays are directly proportional to temperature.

Hence Rise in temperature $=$ temperature + delayed temperature of each nodes

Table: 3 Comparison Temperatures

\begin{tabular}{|c|c|c|}
\hline Time/Nodes & 30 Sec & 300 Sec \\
\hline Node-1 & 0.10 & 2.55 \\
\hline Node-2 & 0.25 & 3.05 \\
\hline Node-3 & 1.00 & 3.25 \\
\hline Node-4 & 1.30 & 4.15 \\
\hline Node-5 & 1.40 & 6.10 \\
\hline
\end{tabular}

A huge difference between the minimum and maximum temperature value with respect to time was observed. Due to human intervention, there might be delays to control the temperature. For example, if delay is 30 seconds on Node1, the temperature is raining to $0: 1 \mathrm{C}$ and if the same is about $300 \mathrm{sec}$ delays and the Temperature (T) rose to 2:55C.We considered the reading for every 30 Seconds. Similarly, all the observations are shown in the Table: 3 .

With the above, it is analysed that human or manual control is slower than the automization process. Any delay will lead to catastrophic damage. 
International Journal of Network Security \& Its Applications (IJNSA), Vol.5, No.1, January 2013

\section{SUMMARY OF THE WORK}

In this paper, we describe an integrated automatic temperature monitoring and controlling system using wireless sensor networks for distributed AC system. The study also describes about the existing centralized system for manual monitoring and controlling as separate or as a whole.

The concept describes about understanding the real time experiments in the data centre such as high performance computing environments. With our prototype we demonstrated the proposed system architecture can effectively satisfy the needs of any data centre or high performance computing environment with distributed AC systems. We also believe that wireless sensor networks could provide and optimal solution for the existing and futuristic conditions. The experimental study on the automization included the daily temperature levels, manual control and its impact with the control and automization.

\section{CONCLUSIONS WITH FUTURE WORK}

In our experiment, we used the common gateway which is a serial connector and limited to control single air conditioning system. In future, it could be utilized with various wireless technologies such as Bluetooth for full pledged solution for different distributed or high performance environment like Grid computing, electrical control rooms/server room etc.

\section{ACKNOWLEDGEMENTS}

We are grateful to the Members of Centre for Development of Advanced Computing (C-DAC), Hyderabad for providing their technical encouragement and supporting our efforts in the case study for providing a optimized solution for integrated automated temperature monitoring and controlling system.

\section{REFERENCES}

[1] Jiang Yi, Qin Xuzong. Cooling metering and charging device in the fan coil unit air conditioning system, HEATING VENTILATION AIR CONDITIONING,,2000,30(6), pp.48-55.

[2] Yang Haichen, Cooling energy metering in the air conditioning system in Shanghai Xianxia Tennis Centre. HEATING VENTILATION \& AIR CONDITIONING , ,2001,31(5), pp.39-42.

[3] Woosung Choi,Woojong and Sangchul, Development of automatic temperature control system in blast furnace, SICE- ICASE,2006.International Joint Conference.

[4] S. V. Srikanth, Pramod P. J, Dileep K. P, Tapas S, Mahesh U. Patil and Sarat Chandra Babu N, Centre for Development of Advanced Computing (C-DAC)Design and Implementation of a prototype Smart PARKing (SPARK) System using Wireless Sensor Networks.

[5] Yao Ye, Hu Yixiong, Xu Xiangbo. Preliminary Research on Calculating Method of Cooling Load in Air-Conditioned Residence,J. Building Energy Environment,2001, pp.8-50.

[6] http://www.sensor-networks.org/index.php?language=english\&page=the group

[7] Sridevi Veerasingam, Saurabh Karodi, Sapna Shukla, Mehar Chaitanya Yeleti Department of Instrumentation and Control Engineering, National Institute of Technology, Tiruchirappalli India, Design of Wireless Sensor Network node on ZigBee for Temperature Monitoring

[8] Ahmad Faris Bin Zulkifli, Automatic Room Temperature Control with Security System.

[9] Vongsagon Boonsawat, Jurarat Ekchamanonta,Kulwadee Bumrungkhet, and Somsak Kittipiyakul School of Information,Computer, and Communication Technology, Thani, Thailand, XBee Wireless Sensor Networks for Temperature Monitoring.

[10] Xiaofang Li,Yuntao Yu,Qingdao University of Science and Technology Qingdao, Shandong Province, China, A High Accuracy Temperature Control System Based on ARM9. 
International Journal of Network Security \& Its Applications (IJNSA), Vol.5, No.1, January 2013

[11] http://www.extremecircuits.net/2010/04/ir-infrared-detector-circuit-diagram.html

[12] http://www.openautomation.net/page/productos/id/22/title/MICAz-2.4-GHz

[13] http://aopen.jp/tech/techinside/bluetooth.html

[14] http://en.wikipedia.org/wiki/First law of thermodynamics

[15] M. Veeraraghavan, X. Zheng, and Z. Huang, On the use of connection-oriented networks to support grid computing, IEEE Commun.Mag., vol. 44, no. 3, pp. 118123, Mar. 2006.

[16] Generalized multi-protocol label switching (GMPLS) signalling functional description, Jan.2003

[17] T.Zhang, K. Lu, and J. P. Jue, Differentiated contention resolution for QoS in photonic packetswitched networks.

[18] http://public.web.cern.ch/Public/Welcome.html

[19] Xiujie Dong, Yan Yang, School of Electric and Information Engineering, Zhongyuan University of Technology China, Study on Household Metering and Temperature Control in Central Airconditioning.

[20] http://ceaccp.oxfordjournals.org/content/8/3/104.full.pdf

[21] http://www.freepatentsonline.com/6956516.html

[22] http://www.hotfrog.com.au/Companies/Abacus-Instruments/Wireless-Temperature-MonitoringSystem-181514

[23] Wang, Wu Chunyu, based on ZigBee technology for remote water meter reading system ,Microprocessors, 2009.6,3, pp.106-107

[24] EPRI, Technical and system requirements of advanced distribution automation.

[25] Lei Yuan, Xiong construction, Zhao Xiaohui.Wi-Fi-based wireless sensor network design andresearch. Modern electronic technology, Nov.2009, vol.18, pp.192-197

[26] Wei Dongjie. Discussion on the metering system of the centralized air conditioning system.Coal Engineering,2003, pp.57-58.

[27] I.J.Gyongy,D.W.Clarke,On the automatic tuning ang adaptation of PID controllers, ControlEngineering Practice.2006.14.149-163.

[28] Li Yu, Huanjia Lu, Zhen Hong from college of Information Engineering, China, Design of Building Monitoring systems based on Wireless Sensors Network by Qifen Dong

\section{Authors}

Working in CDAC for last five years. Working in the field of Network and System Security. Interested areas are Embedded system and Open source.

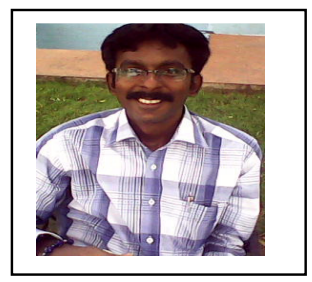

